\title{
Avaliação da autopercepção de saúde de usuários com Diabetes - um estudo de base populacional
}

\author{
Assessment of self-perceived health in users with Diabetes - a population-based study \\ Evaluación de la salud autopercibida en usuarios con diabetes: un estudio poblacional
}

Recebido: 06/09/2021 | Revisado: 12/09/2021 | Aceito: 14/09/2021 | Publicado: 16/09/2021

\author{
Mariana do Couto Soares \\ ORCID: https://orcid.org/0000-0002-8860-4874 \\ Universidade do Vale do Rio dos Sinos, Brasil \\ E-mail: mdocoutosoares@gmail.com \\ Andreia Turmina Fontanella \\ ORCID: https://orcid.org/0000-0003-0455-9429 \\ Universidade Federal do Rio Grande do Sul, Brasil \\ E-mail andreiafontanella@gmail.com \\ Luiza Maria Gerhardt \\ ORCID: https://orcid.org/0000-0003-1558-3811 \\ Universidade Federal do Rio Grande do Sul, Brasil \\ E-mail: luizam1928@gmail.com \\ Maria Luiza Paz Machado \\ ORCID: https://orcid.org/0000-0002-7972-2457 \\ Universidade Federal do Rio Grande do Sul, Brasil \\ E-mail: luiza@enf.ufrgs.br \\ Ana Karina Rocha da Silva Tanaka \\ ORCID: https://orcid.org/0000-0003-2488-3656 \\ Universidade Federal do Rio Grande do Sul, Brasil \\ E-mail: anakarinatanaka@gmail.com
}

\begin{abstract}
Resumo
Objetivo: Descrever os fatores associados a uma autopercepção de saúde em diabéticos de acordo com os resultados de um inquérito populacional de âmbito nacional. Métodos: Utilizamos os dados da Pesquisa Nacional sobre o Acesso, Utilização e Promoção do Uso Racional de Medicamentos (PNAUM), um inquérito populacional de abrangência nacional realizado entre setembro de 2013 a fevereiro de 2014. Resultados: Foi observado uma pior autopercepção de saúde nos diabéticos que: possuíam 3 ou mais DC representando 14,9\%(IC95\%: 12,6-17,5), do que os que apresentavam apenas 1 DC 3,7\% (IC95\%:1,7-7,8); Possuíam outras doenças crônicas como AVC 21,3\%(IC95\%:14,1-30,9), Doença articular crônica 17,8\%(IC95\%:13,4-23,3) e Depressão 24,0\%(IC95\%:18,1-31,1); Possuíam qualquer limitação em decorrência da diabetes e que procuraram o serviço de emergência 30,5\% (IC95\%:21,4-41,5) e passaram por internações hospitalares 20,80\% (IC95\%:14,2- 29,3) nos últimos 12 meses em decorrência da Diabetes Conclusão: Aqueles usuários que apresentaram 3 Doenças Crônicas ou mais, outras doenças crônicas, possuíam qualquer limitação devido ao diabetes e tiveram atendimento no serviço de saúde em decorrência da doença, avaliaram pior sua autopercepção de saúde. A utilização da avaliação da autopercepção de saúde dos usuários durante as consultas com os profissionais, pode ser utilizada como uma ferramenta na estruturação do plano terapêutico direcionado. Por ser um indicador de desfechos de saúde, a autopercepção de saúde pode ser avaliada em conjunto com o contexto pessoal e individual de cada paciente, para que assim possamos realizar um cuidado em saúde mais integral e direcionado a cada paciente.
\end{abstract}

Palavras-chave: Autoimagem; Diabetes Mellitus; Doenças crônicas não transmissíveis.

\begin{abstract}
Objective: To describe the factors associated with self-perceived health in diabetics according to the results of a nationwide population survey. Methods: We used data from the National Survey on Access, Use and Promotion of the Rational Use of Medicines (PNAUM), a nationwide population survey conducted from September 2013 to February 2014. Results: A worse self-perception of health was observed among diabetics who: had 3 or more CD representing 14.9\% (95\%CI: 12.6-17.5), than those who had only 1 CD 3.7\% (95\%CI: 1.7-7.8) ; They had other chronic diseases such as CVA 21.3\% (95\%CI: 14.1-30.9), Chronic joint disease 17.8\% (95\%CI: 13.4-23.3) and Depression 24.0\%(CI95 \%:18.1-31.1); They had any limitation due to diabetes and who sought the emergency service 30.5\% (95\% CI: 21.4-41.5) and underwent hospital admissions 20.80\% (95\%CI: 14.2-29.3) in the last 12 months due to Diabetes Conclusion: Those users who had 3 Chronic Diseases or more, other chronic diseases, had any limitation due to diabetes and had health service care due to the disease, rated their self-perception of health worse. The use of users' self-perceived health assessment during consultations with professionals can be used as a tool in structuring the targeted therapeutic plan. As it is an indicator of health outcomes, self-perception of health can be assessed in
\end{abstract}


conjunction with the personal and individual context of each patient, so that we can provide more comprehensive and targeted health care for each patient.

Keywords: Self-image; Diabetes Mellitus; Chronic non-communicable diseases.

\begin{abstract}
Resumen
Objetivo: Describir los factores asociados a la salud autopercibida en diabéticos según los resultados de una encuesta poblacional a nivel nacional. Métodos: se utilizaron datos de la Encuesta Nacional de Acceso, Uso y Promoción del Uso Racional de Medicamentos (PNAUM), encuesta poblacional a nivel nacional realizada entre septiembre de 2013 y febrero de 2014. Resultados: Se observó una peor salud autopercibida en la población. diabéticos que: tenían 3 o más EC que representan el 14,9\% (IC del 95\%: 12,6-17,5), que los que tenían solo 1 EC 3,7\% (IC del 95\%: 1,7-7,8); Tenían otras enfermedades crónicas como ACV 21,3\% (IC 95\%: 14,1-30,9), enfermedad articular crónica 17,8\% (IC 95\%: 13,4-23,3) y depresión 24,0\% (IC95\%: 18,1-31,1); Tenían alguna limitación por diabetes y acudieron al servicio de urgencias 30,5\% (IC 95\%: 21,4-41,5) y se sometieron a ingresos hospitalarios 20,80\% (IC 95\%: 14,2-29,3) en los últimos 12 meses por Diabetes Conclusión: Aquellos los usuarios que tenían 3 Enfermedades Crónicas o más, otras enfermedades crónicas, tenían alguna limitación por diabetes y tenían atención en el servicio de salud debido a la enfermedad, calificaron peor su autopercepción de la salud. El uso de la autoevaluación de la salud de los usuarios durante las consultas con los profesionales puede utilizarse como una herramienta para estructurar el plan terapéutico específico. Como indicador de los resultados de salud, la autopercepción de la salud se puede evaluar junto con el contexto personal e individual de cada paciente, de modo que podamos brindar una atención médica más integral y específica para cada paciente.
\end{abstract}

Palabras clave: Auto imagen; Diabetes mellitus; Enfermedades crónicas no transmisibles.

\title{
1. Introdução
}

Doenças crônicas não transmissíveis (DCNT) englobam doenças cardiovasculares, respiratórias crônicas, cânceres e diabetes mellitus. Elas são responsáveis por cerca de $70 \%$ das mortes no mundo, estando em crescimento nos últimos anos ${ }^{1}$. No Brasil, as DCNT têm sido responsáveis por $74 \%$ do total de mortes, sendo que as doenças cardiovasculares foram responsáveis por $28 \%$, as neoplasias por $18 \%$, as doenças respiratórias por $6 \%$ e a diabetes por $5 \%$ (Who,2018)

A Diabetes é um distúrbio metabólico que através de um conjunto de fatores, causam hiperglicemia resultante de um mau funcionamento da ação da insulina, na secreção da mesma, ou em ambas. É um grave problema de saúde pública, visto que suas complicações afetam o cotidiano de seus portadores, gerando danos sistêmicos a longo prazo e modificando o estilo de vida destes pacientes (Who, 2018; Arruda, et.al., 2015, Guariguata, et.al, 2014)

No ano de 2017 estimou-se que 8,8\% (intervalo de confiança (IC) de 95\%: 7,2 a 11,3\%) da população mundial com idade entre 20 a 79 anos convivia com a doença, totalizando cerca de 424.9 milhões de pessoas. No ano de 2040 , acredita-se que esta projeção será de 9.9\% (intervalo de confiança (IC) de 95\% 7.5-12.7\%), chegando a 628.6 milhões de pessoas convivendo com a Diabetes (Idf, 2017). Em 2018, a prevalência de diabetes foi de 7,7\% (IC95\% 7,2 - 8,1) no Brasil segundo dados da Vigilância de Fatores de Risco para doenças crônicas não transmissíveis (VIGITEL) (Brasil, 2018).

A autopercepção de saúde é uma avaliação do estado de saúde individual de cada usuário. A mesma, já tem sido descrita na literatura como uma medida válida, confiável, robusta do estado de saúde e de fácil obtenção. Ela avalia a percepção de saúde com uma simples pergunta, e reflete a forma como o usuário enxerga sua saúde naquele momento de sua vida (Medeiros, et.al., 2016).

Estudos trazem que a percepção de saúde pode prever mortalidade, autocuidado e um declínio funcional, sendo citada como um importante indicador do impacto das doenças crônico degenerativas, assim como um potente indicativo do bem-estar físico, social e mental dos pacientes (Arruda,et.al., 2015, Vejem,et.al., 2017)

A autopercepção de saúde pode ser influenciada por fatores biopsicossociais, refletindo os diversos contextos nos quais os usuários encontram-se inseridos. Conhecer alguns destes fatores, pode auxiliar o profissional de saúde na hora de direcionar o cuidado de forma mais individualizada, atingindo pontos específicos e próprios do usuário auxiliando em um tratamento de maior sucesso (Vejem, et.al., 2017). 
Com o envelhecimento e aumento da longevidade da população, há o aparecimento de doenças crônicas e incapacidades funcionais derivadas das mesmas, que podem afetar as condições de saúde da população. Cada vez mais, são necessárias o desenvolvimento de pesquisas epidemiológicas para obter dados populacionais, avaliando as políticas públicas de saúde (Rodrigues, et.al., 2018)

Avaliando a importância descrita da autopercepção de saúde em pacientes com doenças crônicas não transmissíveis como a diabetes, vemos que a mesma pode ser uma importante ferramenta para os profissionais da saúde utilizarem durante a realização do cuidado e escolha da melhor abordagem terapêutica. Sendo assim, este estudo tem como objetivo, descrever os fatores associados a autopercepção de saúde dos diabéticos de acordo com os resultados de um inquérito populacional de âmbito nacional.

\section{Metodologia}

A Pesquisa Nacional sobre Acesso, Utilização e Promoção do Uso Racional de Medicamentos no Brasil (PNAUM), foi uma pesquisa instituída na Portaria nº 2.077, de 17 de Setembro de 2012 do Ministério da Saúde. Ela buscou avaliar e fornecer informações às políticas públicas de assistência farmacêutica no Brasil. Nela foram avaliados o acesso e uso dos medicamentos dos usuários e seus familiares, bem como suas comorbidades, investimentos na área e retorno dos investimentos realizados A escolha da metodologia de pesquisa, deu-se através de estudos baseados em Koche, 2011 e Pereira, et.al., 2018 (Brasil, 2016)

A PNAUM foi composta por diversos setores do Ministério da saúde, pesquisadores da Fundação Oswaldo Cruz e diversas Universidades Federais do País. Para sua execução, a PNAUM obteve a separação em duas grandes áreas, sendo elas:1) Componente populacional (inquérito domiciliar sobre acesso, utilização e uso racional de medicamentos) e a 2) Componente serviços (avaliação das políticas públicas de assistência farmacêutica e sua efetivação na Atenção Básica à Saúde no SUS) (Gadelha, et.al., 2016, Mengue,et.al., 2016)

A PNAUM serviços teve como objetivo avaliar as políticas públicas farmacêuticas na Atenção Básica do País, através dos serviços de saúde. Esta parte do desenvolvimento da pesquisa ficou com a Universidade Federal de Minas Gerais. Para o desenvolvimento da pesquisa foram consideradas como população de estudo: secretários municipais de saúde, responsáveis pela assistência farmacêutica nos municípios, dispensadores de medicamentos, médicos e usuários dos serviços de saúde, sendo que foram sorteados 120 municípios em cada região (Alvares, et.al., 2017)

Na PNAUM inquérito, foram mais de 40 mil moradores entrevistados em domicílios da zona urbana, nos 26 municípios da Federação do País mais o Distrito Federal. O inquérito domiciliar da PNAUM teve por objetivo avaliar a utilização de medicamentos pela população brasileira, com a caracterização das morbidades para as quais eles são utilizados, indicadores de acesso e racionalidade do uso de medicamentos segundo variáveis demográficas, socioeconômicas, de estilo de vida e de morbidade. Dentro deste inquérito, a última pergunta referia-se a percepção de saúde do entrevistado no momento (Mengue, et.al., 2016) ${ }^{13}$

A PNAUM foi um estudo de abordagem quantitativa transversal de caráter analítico de dados de base populacional, desenvolvida entre setembro de 2013 a outubro de 2014.

Para delimitar a estimativa do tamanho amostral, as informações preliminares da população, foram obtidas a partir da análise dos dados de acesso aos medicamentos, publicados na Pesquisa Nacional por Amostra de Domicílios (Pnad) do ano de 2008, do Instituto Brasileiro de Geografia e Estatística (IBGE). A população total do estudo foi de 41.433 pessoas entrevistadas. 
No estudo de Mengue et.al. 2016, os autores descrevem em detalhes toda a metodologia utilizada no inquérito domiciliar da PNAUM. Neste estudo entende-se melhor o desenho utilizado no estudo, processo amostral, instrumentos elaborados para coleta de dados e a implementação no campo de coleta da PNAUM inquérito.

Para o desenvolvimento desta pesquisa, analisamos através de uma amostra probabilística o banco de dados da PNAUM que encontra-se disponível online através do site (http://www.ufrgs.br/pnaum). Nesta pesquisa, foram selecionando os entrevistados adultos, capazes de se comunicar, com 20 anos ou mais e que possuíam o diagnóstico médico autorreferido de diabetes. A amostra para a realização deste trabalho totalizou 2494 entrevistados.

Como variáveis do estudo avaliamos o sexo (feminino e masculino), faixa etária (20 a 39 anos, 40 a 59 anos e 60 anos ou mais), , escolaridade (nunca estudou, 1 a 8 anos de estudo completo e mais de 8 anos de estudo completos), regiões do brasil (norte, nordeste, sudeste, sul e centro oeste), número de doenças crônicas (nenhuma, uma, duas e três ou mais), classe social segundo escore ABEP ( $\mathrm{A} / \mathrm{B}, \mathrm{C}$ e $\mathrm{D} / \mathrm{E}$ ), prevalência da autopercepção de saúde (muito ruim/ruim, regular, muito bom/bom), procura pelos serviços de saúde (acesso ao serviço de emergência e internações hospitalares nos últimos 12 meses), limitações relacionadas ao diabetes (sem limitações, poucas, moderadamente, intensamente e muito intensamente) e outras doenças crônicas além da diabetes (hipertensão, AVC, doença articular crônica e depressão). Foram apresentadas as frequências relativas de cada categoria das variáveis e em cruzamento com a autopercepção de saúde, sendo todos os resultados apresentados com o intervalo de confiança de $95 \%$.

Para a análise bivariada entre variáveis categóricas foram utilizado o teste Qui-quadrado de Pearson de igualdade de proporções. Os dados foram analisados no programa IBM SPSS versão 18.0 para Windows, sendo que, para critérios de decisão estatística adotou-se o nível de significância de 5\%.

A Pesquisa Nacional sobre Acesso, Utilização e Promoção do Uso Racional de Medicamentos (PNAUM) foi submetida à Comissão Nacional de Ética Em Pesquisa (Conep), sob o Certificado de Apresentação para Apreciação Ética (CAAE) 18947013.6.0000.0008, e foi aprovada mediante o parecer n 398.131/2013, para execução em âmbito nacional.

Para os entrevistados durante o inquérito domiciliar da PNAUM foi realizada uma breve descrição sobre os objetivos e propósitos da pesquisa e como seriam realizadas as coletas de dados. As entrevistas foram realizadas entre o período de setembro de 2013 a outubro de 2014 e ocorreram após a assinatura do Termo de Consentimento Livre e Esclarecido (TCLE), cada entrevistado ficou com um cópia do seu termo assinado. Os resultados desta pesquisa foram divulgados com a garantia de anonimato dos participantes, e encontram-se disponíveis gratuitamente.

Os dados da PNAUM são dados públicos, e encontram-se disponíveis de acordo com o previsto segundo a Lei de Acesso de Informação - Lei n 12.527 , de 18 de Novembro de 2011 e Decreto nº 8.777, de 11 de Maio de 2016.

\section{Resultados}

No quadro 1 são descritas as características da população total do estudo da amostra selecionada do banco de dados. A prevalência de diabetes na população do estudo foi de 6,8\% (IC95\% 6,4-7,2), sendo que a maioria da população eram do sexo feminino $(53,8 \%)$, e destas 7,7\% (IC95\% 7,2-8,3) se autodeclararam com diabetes. Na população masculina (46,2\%), s 5,3\% (IC95\% 4,8-5,9) eram diabéticos autodeclarados. Ao avaliar a faixa etária da população do estudo 45,7\% possuíam de 20 a 39 anos, 36,2\% de 40 a 59 anos e 18,1\% eram maiores de 60 anos. Já a população com diabetes autorreferido, 1,1\% (IC95\% 0,9-1,4) possuíam de 20 a 39 anos, 7,4\%c (IC95\% 6,8-8,1) tinham entre 40 e 59 anos e 18,9\% (IC95\% 17,8-20) eram maiores de 60 anos.

Quanto a análise da escolaridade, observou-se que 13,9\% do total dos usuários nunca haviam estudado, e destes, 6,6\% (IC95\% 5,7-7,7) possuíam o diagnóstico de diabetes autorreferido. Daqueles que referiram escolaridade de 1 a 8 anos $(43,4 \%)$, 
6,4\% (5,9-7) possuíam o diagnóstico autorreferido de diabetes, sendo que aqueles usuários que possuíam mais de 8 anos de estudo (42,1\%), 6,8\% (IC95\% 6,1-7,5) possuíam o diagnóstico autorreferido de diabetes.

Ao avaliar por regiões do Brasil, o sudeste aparece em primeiro lugar representando 47,7\% do total dos usuários, sendo 7,4\% (IC95\% 6,6-8,2) desta população com diabetes, seguido pelo nordeste $(23,1 \%$ ), sendo que 5,6\% (IC95\% 5-6,2) destes possuíam diagnóstico de diabetes. No sul (14,7\%), sendo destes 6,8\% (IC95\% 6,1-7,5) com diagnóstico de diabetes. O centro-oeste totalizou 7,8\% dos usuários, e destes, 6,5 (IC95\% ,8-7,3) eram portadores de diabetes, e o norte aparece como último lugar representando 6,7\% do total de usuários da pesquisa, sendo 4,3\% (IC95\% 3,7-4,9) destes portadores de diabetes.

Ao utilizar a classificação do Escore da ABEP para classes econômicas foram dividas em 3 categorias, sendo elas: classe A/B com maior renda tendo a representação de 24,3\% do total dos usuários, destes 6,5\% (IC95\% 5,8-7,3) possuíam o diagnóstico autorreferido de diabetes; Classe C, intermediária, representando $55,1 \%$ do total dos usuários, e destes $6,6 \%$ (IC95\% 6,1-7,7) possuíam diagnóstico autorreferido de diabetes; Classe D/E representando 20,5\% do total de usuários, sendo $6,8 \%$ (IC95\% 6,1-7,7) destes portadores de diabetes.

Foi avaliada o número de doenças crônicas (DC) que os usuários possuíam. Cerca de $61 \%$ deles responderam que não possuíam nenhuma doença crônica, 20,1\% responderam que possuíam apenas 1 DC, e destes 5,2\% (4,5-6) possuíam o diagnóstico autorreferido de diabetes; 9,8\% responderam que possuíam 2 DC sendo que 20,2\% (18,4-22,2) tinham o diagnóstico autorreferido de diabetes e 9,1\% disseram ter 3 DC ou mais, sendo que 39,6\% (36,9-42,3) tinham diabetes.

$\mathrm{Na}$ avaliação da autopercepção de saúde dos usuários, utilizamos como base a última pergunta do questionário (ANEXO 1) “Em geral, como o(a) Sr(a) avalia sua saúde?”. Para análise foi dividido em 3 categorias, sendo elas: Muito boa/boa representado por 47,6\% do total de usuários, 3,7\% (3,4-4,1) destes possuíam o diagnóstico autorreferido de diabete; Regular representando $22,1 \%$ do total de usuários, sendo $14 \%(12,8-15,2)$ diabéticos; Muito ruim/ruim representando apenas $3,4 \%$ do total dos usuários, $21,6 \%(18,9-24,6)$ destes eram diabéticos.

Quadro 1 - Caracterização da população estudada (Continua).

\begin{tabular}{|c|c|c|c|}
\hline \multirow[t]{2}{*}{ Caracterização da amostra } & Total & \multicolumn{2}{|c|}{ Com DM } \\
\hline & $\%$ & $\%$ & IC $95 \%$ \\
\hline Sexo & \multicolumn{3}{|c|}{$\mathrm{p}<0,001$} \\
\hline Feminino & $\mathbf{5 3 . 8}$ & 7.7 & {$[7,2-8,3]$} \\
\hline Masculino & 46.2 & 5.3 & {$[4,8-5,9]$} \\
\hline Faixa etária & \multicolumn{3}{|c|}{$\mathrm{p}<0,001$} \\
\hline $20-39$ anos & 45.7 & 1.1 & {$[0,9-1,4]$} \\
\hline $40-59$ anos & 36.2 & 7.4 & {$[6,8-8,1]$} \\
\hline$>60$ anos & 18.1 & 18.9 & {$[17,8-20,0]$} \\
\hline Escolaridade & \multicolumn{3}{|c|}{$\mathrm{p}<0,677$} \\
\hline Nunca estudou & 13.9 & 6.6 & {$[5,7-7,7]$} \\
\hline
\end{tabular}




\begin{tabular}{|c|c|c|c|}
\hline 1 a 8 anos de estudo & 43.4 & 6.4 & {$[5,9-7,0]$} \\
\hline Mais de 8 anos de estudo & 42.1 & 6.8 & {$[6,1-7,5]$} \\
\hline Regiões do Brasil & \multicolumn{3}{|c|}{ p $<0,001$} \\
\hline Norte & 6.7 & 4.3 & {$[3,7-4,9]$} \\
\hline Nordeste & 23.1 & 5.6 & {$[5,0-6,2]$} \\
\hline Sudeste & 47.7 & 7.4 & {$[6,6-8,2$} \\
\hline Sul & 14.7 & 6.8 & {$[6,1-7,5]$} \\
\hline Centro - Oeste & 7.8 & 6.5 & {$[5,8-7,3]$} \\
\hline Doenças Crônicas & \multicolumn{3}{|c|}{$\mathrm{p}<0,001$} \\
\hline Nenhuma & 61 & & \\
\hline 1 & 20.1 & 5.2 & {$[4,5-6,0]$} \\
\hline 2 & 9.8 & 20.2 & {$[18,4-22,2]$} \\
\hline 3 ou mais & 9.1 & 39.6 & {$[36,9-42,3]$} \\
\hline Escore ABEP (renda) & \multicolumn{3}{|c|}{$\mathrm{p}<\mathbf{0 , 8 1 2}$} \\
\hline $\mathbf{A} / \mathbf{B}$ & 24.3 & 6.5 & {$[5,8-7,3]$} \\
\hline $\mathbf{C}$ & 55.1 & 6.6 & {$[6,1-7,7]$} \\
\hline $\mathbf{D} / \mathbf{E}$ & 20.5 & 6.8 & {$[6,1-7,7]$} \\
\hline Autopercepção em saúde & \multicolumn{3}{|c|}{$\mathrm{p}<0,001$} \\
\hline Muito bom / bom & 47.6 & 3.7 & {$[3,4-4,1]$} \\
\hline Regular & 22.1 & 14 & {$[12,8-15,2]$} \\
\hline Ruim/ Muito Ruim & 3.4 & 21.6 & {$[18,9-24,6]$} \\
\hline
\end{tabular}

Fonte: Autores.

Para avaliação dos fatores que influenciavam na autopercepção de saúde dos usuários, nós selecionamos apenas os adultos maiores de 20 anos, capazes de se comunicar e que tenham autodeclarado o diagnóstico médico de diabetes.

No quadro 2 avaliamos o número de doenças crônicas associado à autopercepção dos usuários que possuíam o diagnóstico autorreferido de diabetes. Usuários que possuíam 1 Doença Crônica (DC) relataram que 64,80\% (57,9-71,1) possuíam avaliação de autopercepção de saúde Muito boa/boa, 31,5\% (25,4-38,2) Regular e 3,7\% (1,7-7,8) Muito ruim/ruim. Aqueles que possuíam 2 DC, 47,7\% (42,7-52,8) relataram ter uma Muito bom/bom autopercepção de saúde, 44,5\% (39,7-49,5) 
relataram ter uma autopercepção Regular e 7,8\% (5,6-10,8) relataram ter uma autopercepção Muito ruim/ruim de sua saúde. Já aqueles que possuíam o diagnóstico médico autorreferido de diabetes e adicionado a isso possuíam 3 ou mais DC, destes $32,7 \%(29,3-36,3)$ ter uma Muito bom/bom autopercepção, 52,5\% (49-55,9) relataram ter uma autopercepção Regular e, 14,9\% (12,6-17,5) relataram uma autopercepção Muito ruim/ruim.

Quadro 2 - Associação da autopercepção de saúde com número de doenças crônicas.

\begin{tabular}{|c|c|c|c|c|c|c|}
\hline \multirow[t]{4}{*}{ Doenças Crônicas } & \multicolumn{6}{|c|}{ Autopercepção de saúde } \\
\hline & \multicolumn{6}{|c|}{ p $<0,001$} \\
\hline & \multicolumn{2}{|c|}{ Muito bom/bom } & \multicolumn{2}{|c|}{ Regular } & \multicolumn{2}{|c|}{ Muito ruim/ruim } \\
\hline & $\%$ & IC $95 \%$ & $\%$ & IC $95 \%$ & $\%$ & IC $95 \%$ \\
\hline $1 \mathrm{DC}$ & $64.80 \%$ & {$[57,9-71,1]$} & $31.50 \%$ & {$[25,4-38,2]$} & $3.70 \%$ & {$[1,7-7,8]$} \\
\hline 2 DC & $47.70 \%$ & {$[42,7-52,8]$} & $44.50 \%$ & {$[39,7-49,5]$} & $7.80 \%$ & {$[5,6-10,8]$} \\
\hline 3 ou + DC & $32.70 \%$ & {$[29,3-36,3]$} & $52.50 \%$ & {$[49-55,9]$} & $14.90 \%$ & {$[12,6-17,5]$} \\
\hline
\end{tabular}

Fonte: Autores.

Para avaliar quais seriam as outras doenças crônicas que os usuários possuíam além do diabetes, realizamos a associação separadamente destas outras doenças com a autopercepção de saúde. No quadro 3, ao avaliarmos a Hipertensão $38,1 \%$ (35-41,4) dos usuários que possuem esta doença além do diabetes, relatou uma percepção Muito bom/bom. 49,\% (46,853,1) destes relataram uma percepção Regular e $12 \%$ disseram ter uma percepção Muito ruim/ruim. Aqueles usuários que não possuíam hipertensão 53,2\% (47,6-58,6) relataram ter uma percepção Muito boa/boa, 38,4\% $(33,3-43,9)$ Regular e 8,4\% (5,811,9) Muito ruim/ruim.

Na avaliação dos usuários que possuíam AVC 27,6\% (19,4-37,6) relataram uma percepção Muito bom/bom, 51,1\% (40,8-61,3) Regular e 21,3\% (14,1-30,9) Muito ruim/ruim. Dos usuários que não possuíam o diagnóstico de AVC 43,4\% (40,446,4) relataram uma percepção Muito boa/boa, 46,4\% (43,7-49,2) Regular e 10,2\% (8,6-12) referiram uma percepção Muito ruim/ruim.

Ao avaliar os usuários que possuíam doença articular crônica 29,3\% (23,9-35,5) referiram uma percepção Muito bom/bom, 52,9\% (46,8-58,8) Regular e 17,8\% relataram uma percepção Muito ruim/ruim. Daqueles que referiram não ter o diagnóstico de doença articular crônica $45 \%$ (41,8-48,2) relataram percepção Muito bom/bom, 45,5\% (42,4-48,6) Regular e 9,50\% (8-11,4) Muito ruim/ruim.

Na avaliação dos usuários que possuíam diagnóstico de depressão 29\% (22,2-37) avaliaram sua percepção como Muito boa/boa, 46,9\% (40-54) Regular e 24\% (18,1-31,1) Muito ruim/ruim. Aqueles que não possuíam depressão 43,9\% (40,947,1) avaliaram percepção como Muito boa/boa, 46,8\% (43,8-49,7) Regular e 9,3\% (7,8-11) como Muito ruim/ruim. 
Quadro 3 - Associação da autopercepção de saúde com outras doenças crônicas.

\begin{tabular}{|c|c|c|c|c|c|c|}
\hline \multirow[t]{3}{*}{ Outras Doenças Crônicas } & \multicolumn{6}{|c|}{ Autopercepção de saúde } \\
\hline & \multicolumn{2}{|c|}{ Muito bom/bom } & \multicolumn{2}{|c|}{ Regular } & \multicolumn{2}{|c|}{ Muito ruim/ruim } \\
\hline & $\%$ & IC $95 \%$ & $\%$ & IC $95 \%$ & $\%$ & IC $95 \%$ \\
\hline Hipertensão & \multicolumn{6}{|c|}{$\mathrm{p}<\mathbf{0 , 0 0 1}$} \\
\hline Sim & $38.10 \%$ & {$[35-41,4]$} & $49.90 \%$ & {$[46,8-53,1]$} & $12 \%$ & {$[10,3-13,9]$} \\
\hline Não & $53.2 \%$ & {$[47,6-58,6]$} & $38.40 \%$ & {$[33,3-43,9]$} & $8.40 \%$ & {$[5,8-11,9]$} \\
\hline AVC & \multicolumn{6}{|c|}{$\mathrm{p}<0,001$} \\
\hline Sim & $27.60 \%$ & {$[19.4-37,6]$} & $51.10 \%$ & {$[40,8-61,3]$} & $21.30 \%$ & {$[14,1-30,9]$} \\
\hline Não & $43.40 \%$ & {$[40,4-46,4]$} & $46.40 \%$ & {$[43,7-49,2]$} & $10.20 \%$ & {$[8,6-12]$} \\
\hline Doença articular crônica & \multicolumn{6}{|c|}{$\mathrm{p}<0,001$} \\
\hline Sim & $29.30 \%$ & {$[23,9-35,5]$} & $52.90 \%$ & {$[46,8-58,8]$} & $17.80 \%$ & {$[13,4-23,3]$} \\
\hline Não & $45.00 \%$ & {$[41,8-48,2]$} & $\mathbf{4 5 . 5 0 \%}$ & {$[42,4-48,6]$} & $9.50 \%$ & {$[8-11,4]$} \\
\hline Depressão & \multicolumn{6}{|c|}{$\mathrm{p}<0,001$} \\
\hline Sim & $29.00 \%$ & {$[22,2-37]$} & $46.90 \%$ & {$[40-54]$} & $24.00 \%$ & {$[18,1-31,1]$} \\
\hline Não & $43.90 \%$ & {$[40,9-47,1]$} & $46.80 \%$ & {$[43,8-49,7]$} & $9.30 \%$ & {$[7,8-11]$} \\
\hline
\end{tabular}

Fonte: Autores.

No quadro 4, ao avaliarmos as limitações causadas pela Diabetes associadas a autopercepção de saúde, observamos que cerca de 49,7\% (46,1-52,3) não possuíam limitações e avaliaram sua autopercepção como muito boa/boa, 44,1\%(40,747,6) usuários sem limitações avaliaram sua autopercepção como regular e 6,2\% (4,8-8,0) avaliou como muito ruim/ruim. Já aqueles usuários que possuíam poucas limitações 31,5\% (25,5-38,3) relataram uma autopercepção muito boa/boa, 52,3\% $(45,8-58,8)$ regular e $16,2 \%(12,6-20,4)$ muito ruim/ruim. Daqueles usuários que possuíam limitações moderadamente 23,6\% (17,5-31,1) relataram autopercepção muito boa/boa, 54,4\% (38,3-62,6) regular e 22,0\% (15,8-29,8) muito ruim/ruim. Ao avaliar usuários que possuíam limitações intensas, 12,6\% (6,5-23,2) avaliaram uma autopercepção muito boa/boa, 50,5\% $(38,2-62,6)$ regular e 36,8\% (26,4-48,7). Já aqueles que possuíam limitações muito intensas, 22,0\% (9,9-42,0) relataram autopercepção muito boa/boa, 46,8\% (27,5-67,1) regular e $31,2 \%(17,0-50,1)$ muito ruim/ruim. 
Quadro 4 - Associação da autopercepção de saúde com limitações causadas pela Diabetes.

\begin{tabular}{|c|c|c|c|c|c|c|}
\hline \multirow[t]{4}{*}{ Limitação DM } & \multicolumn{6}{|c|}{ Autopercepção de saúde } \\
\hline & \multicolumn{6}{|c|}{$\mathrm{p}<0,001$} \\
\hline & \multicolumn{2}{|c|}{ Muito bom/bom } & \multicolumn{2}{|c|}{ Regular } & \multicolumn{2}{|c|}{ Muito ruim/ruim } \\
\hline & $\%$ & IC $95 \%$ & $\%$ & IC $95 \%$ & $\%$ & IC $95 \%$ \\
\hline Sem limitações & $49.7 \%$ & {$[46,1-53,3]$} & $44.1 \%$ & {$[40,7-47,6]$} & $6.2 \%$ & {$[4,8-8,0]$} \\
\hline Poucas & $31.5 \%$ & {$[25,5-38,3]$} & $52.3 \%$ & {$[45,8-58,8]$} & $16.2 \%$ & {$[12,6-20,4]$} \\
\hline Moderadamente & $23.6 \%$ & {$[17,5-31,1]$} & $54.4 \%$ & {$[45,9-62,6]$} & $22.0 \%$ & {$[15,8-29,8]$} \\
\hline Intensamente & $12.6 \%$ & {$[6,5-23,2]$} & $50.5 \%$ & {$[38,3-62,6]$} & $36.8 \%$ & {$[26,4-48,7]$} \\
\hline Muito intensamente & $22.0 \%$ & {$[9,9-42]$} & $46.8 \%$ & {$[27,5-67,1]$} & $31.2 \%$ & {$[17,0-50,1]$} \\
\hline
\end{tabular}

Fonte: Autores.

No quadro 5 observamos a procura pelos serviços de saúde, avaliados pelo atendimento em emergência e hospitalar nos últimos 12 meses. Aqueles usuários que procuraram o serviço de emergência, 32,7\% (27,4-38,5) relataram uma autopercepção muito boa/boa, 49,20\% (43,3-55,1) regular e 18,10\% (14,5-22,4) muito ruim/ruim. Já aqueles usuários que não passaram por este atendimento, 45,80\% (42,6-49,0) relataram uma autopercepção muito boa/boa, 45,90\% $(42,9-48,9)$ regular e $8,30 \%(6,7-10,2)$ muito ruim/ruim.

Na avaliação dos usuários que procuraram atendimento hospitalar 31,60\% (24,9-39,1) relataram uma autopercepção muito boa/boa, 49,50\% (42,1-56,8) regular e 19,0\% (14,4-24,5) muito ruim/ruim. Entre os usuários que não procuraram este serviço, 44,10\% (41,1-47,1) relataram uma autopercepção muito boa/bom, 46,30\% $(43,5-49,2)$ regular e 9,60\% muito ruim/ruim.

$\mathrm{Na}$ avaliação da associação da procura pelos serviços de saúde nos últimos 12 meses por motivos relacionados ao Diabetes, os usuários que procuraram o serviço de emergência por outros motivos avaliaram sua autopercepção como muito bom/bom 42,60\% (39,7-45,7), regular 47,40\% (44,7-50,2) e muito ruim/ruim 9,90\% (8,4-11,7). Aqueles usuários que procuraram os serviços de emergências devido à diabetes 34,90\% (25,2-46,2) avaliaram sua autopercepção como muito bom/bom, 34,60\% (25,3-45,2) como regular e 30,50\% (21,4-41,5) como muito ruim/ruim.

$\mathrm{Na}$ avaliação dos usuários que passaram por internações hospitalares por outros motivos, 43,10\% (40,2-46,1) avaliaram sua autopercepção de saúde como muito bom/bom, 46,50\% (43,8-49,3) como regular e 10,30\% (14,2-26,8) como muito ruim/ruim. Já aqueles usuários que passaram por internações devido a Diabetes, 29,20\% (20,1-40,3) avaliaram sua autopercepção como muito bom/bom, $50 \%(40,2-59,9)$ como regular e 20,8\% (14,2-26,8) como muito ruim/ruim. 
Quadro 5 - Associação da autopercepção de saúde com a procura pelo serviço de saúde e motivos da procura pelos serviços de saúde (Continua).

\begin{tabular}{|c|c|c|c|c|c|c|}
\hline Serviços de saúde & \multicolumn{6}{|c|}{ Autopercepção de saúde } \\
\hline & \multicolumn{6}{|c|}{ p $<0,001$} \\
\hline & \multicolumn{2}{|c|}{ Muito bom/bom } & \multicolumn{2}{|c|}{ Regular } & \multicolumn{2}{|c|}{ Muito ruim/ruim } \\
\hline Atendimento Emergência & $\%$ & IC $95 \%$ & $\%$ & IC $95 \%$ & $\%$ & IC $95 \%$ \\
\hline Sim & $32.70 \%$ & {$[27,4-38,5]$} & $49.20 \%$ & {$[43,3-55,1]$} & $18.10 \%$ & {$[14,5-22,4]$} \\
\hline Não & $45.80 \%$ & {$[42,6-49]$} & $45.90 \%$ & {$[42,9-48,9]$} & $8.30 \%$ & {$[6,7-10,2]$} \\
\hline Atendimento Hospitalar & $\%$ & IC $95 \%$ & $\%$ & IC $95 \%$ & $\%$ & IC $95 \%$ \\
\hline Sim & $31.60 \%$ & {$[24,9-39,1]$} & $49.50 \%$ & {$[42,1-56,8]$} & $19.00 \%$ & {$[14,4-24,5]$} \\
\hline Não & $44.10 \%$ & {$[41,1-47,1]$} & $46.30 \%$ & {$[43,5-49,2]$} & $9.60 \%$ & {$[8-11,4]$} \\
\hline Motivo da procura do serviço de saúde & $\%$ & IC $95 \%$ & $\%$ & IC $95 \%$ & $\%$ & IC $95 \%$ \\
\hline Procura pelo DM - emergências & $34,90 \%$ & {$[25,2-46,2]$} & $34,60 \%$ & {$[25,3-45,2]$} & $\mathbf{3 0 , 5 0 \%}$ & {$[21,4-41,5]$} \\
\hline Procura por outros motivos - emergências & $42,60 \%$ & {$[39,7-45,7]$} & $47,40 \%$ & {$[44,7-50,2]$} & $9,90 \%$ & {$[8,4-11,7]$} \\
\hline Procura pelo DM - internações & $29,20 \%$ & {$[20,1-40,3]$} & $\mathbf{5 0 , 0 0 \%}$ & {$[40,2-59,9]$} & $20,80 \%$ & {$[14,2-29,3]$} \\
\hline Procura por outros motivos - Internações & $43,10 \%$ & {$[40,2-46,1]$} & $46,50 \%$ & {$[43,8-49,3]$} & $10,30 \%$ & {$[14,2-26,8]$} \\
\hline
\end{tabular}

Fonte: Autores.

\section{Discussão}

A prevalência de diabetes encontrada na população do nosso estudo foi de $6,5 \%$, sendo identificada a maior prevalência entre as mulheres 7,7\% (IC95\%:7,2-8,3), do que nos homens 5,3\% (IC95\%:4,8-5,9). Este resultado foi semelhante ao apontado pelos dados da Pesquisa Nacional de Saúde em 2013, na qual cerca de 6,2\% da população referiu o diagnóstico de diabetes (Isler, et.al., 2015).

A maioria dos entrevistados eram do sexo feminino representando 53,8\%, destas, 7,7\% (IC95\%:7,2-8,3) possuíam diabetes. No Brasil segundo a Pesquisa Nacional por Amostra de Domicílios Contínua do Primeiro Trimestre de 2019, a maioria da população brasileira acima dos 14 anos era feminina (Ibge,2019). Em outros estudos, as mulheres também aparecem como maioria (Silva, et.al., 2015, Junior, et.al., 2018). Esta diferença de gênero, demonstra a constante necessidade de implementar políticas públicas direcionadas, reduzindo as diferenças no acesso aos serviços de saúde.

A maioria da população com o diagnóstico de diabetes estavam na faixa etária maior de 60 anos (18,9\% (IC95\%:17,820)), eram da região Sudeste $(7,4 \%(6,6-8,2))$, possuíam 3 doenças crônicas ou mais $(39,6 \%$ (IC95\%:36,9-42,3)), e relataram ter uma pior autopercepção de saúde (21,6\%(IC95\%:18,9-24,6)). A população idosa (> 60 anos), vem crescendo cada vez mais no Brasil. Em 2015, segundo IBGE a população idosa brasileira representava 14,3\%, sendo que a projeção para 2060 é de que esta população aumente para 25,5\%. Em 2018, a população de idosos era de 31.981 mil brasileiros, representando $15,4 \%$ da população total (Ibge, 2019).

Estes dados demonstram que a população brasileira se tornará uma população envelhecida, que possuirá maiores demandas de saúde, muitas vezes relacionadas às doenças crônicas não transmissíveis, como a diabetes, trazendo a importância 
de trabalhar a prevenção e promoção de saúde, oportunizando uma melhor qualidade de vida e autonomia para esta população. Dados como estes auxiliam no desenvolvimento de políticas públicas mais direcionadas, preparando os serviços de saúde para atenderem a esta demanda, trabalhando na desmistificação de que doenças crônicas devem fazer parte do envelhecimento, mostrando a importância de trabalhar a prevenção, promoção e educação em saúde (Junior, et.al., 2018)

Ao avaliar a pior autopercepção de saúde associada com o número de doenças crônicas (DC), 14,9\%(IC95\%:12,617,5) referiram 3 DC ou mais, enquanto que 3,70\% (IC95\%:1,7-7,8) referiram ter 1 DC. Outras doenças crônicas como AVC, Doença articular crônica e Depressão influenciaram negativamente na autopercepção de saúde quando comparados aos usuários que não possuíam estas doenças, representando 21,3\%(IC95\%:14,1-30,9), 17,80\%(IC95\%:13,4-23,3) e 24\%(IC95\%:18,1-31,1) respectivamente. Doenças como diabetes, AVC, depressão e doença articular crônica vem sendo citadas como causadoras de limitações nas atividades diárias dos pacientes, influenciando na autonomia e autopercepção de saúde, sendo que aquelas que causam deficiências funcionais causam maior impacto na percepção de saúde (Boccolini, et.al., 2017, Chiu, et.al., 2017).

Ao observar os resultados referente às doenças crônicas mais prevalentes, a presença de depressão mostrou-se capaz de influenciar na percepção de saúde dos usuários. Tendo em vista que a autopercepção de saúde é uma medida subjetiva, doenças mentais como a depressão afetam a concepção pessoal de cada paciente, influenciando no modo em que ele avalia e enxerga sua saúde. Um estudo de Chiu et.al.2017, descreveu que a avaliação da autopercepção de saúde junto com outros fatores como limitações causadas pelas doenças, influenciam na manifestação de sintomas relacionados ao quadro de depressão. Isto nos faz pensar que, se avaliarmos a percepção de saúde conseguiremos atuar na prevenção da saúde daquele indivíduo, diminuindo os sinais e sintomas da doença melhorando a qualidade de vida daquele paciente (Borges, et.al., 2014)

Quando avaliamos não apenas a presença de doenças crônicas, mas também sua frequência, elas pareceram influenciar negativamente na avaliação da autopercepção de saúde. Resultados semelhantes já haviam sido descritos anteriormente (Cislaghi \& Cislaghi, 2019, Lefevre, et.al., 2014). O envelhecimento da população pode acarretar em um aumento no aparecimento de doenças crônicas não transmissíveis. A multimorbidade é quando os indivíduos apresentam mais de um problema de saúde em diferentes sistemas do organismo, como hipertensão, diabetes e problemas cardiovasculares É uma condição de saúde que vem crescendo sua manifestação ao longo dos anos, e acomete mais os idosos (Calvalcanti, et.al., 2017) A presença deste fator, aumenta o risco de mortalidade, modifica estilos de vida e provoca um impacto na saúde destes indivíduos, modificando sua percepção de saúde por afetar fatores sociais, físicos e mentais, estando associada a uma avaliação negativa da autopercepção de saúde (Fabbri, et.al., 2015, Dias,et.al., 2016) Com o envelhecimento progressivo da população, devemos nos atentar a fatores como multimorbidade para realizarmos intervenções de saúde que possam auxiliar a população, utilizando a avaliação da autopercepção de saúde como um guia para promover um melhor tratamento, direcionando o cuidado.

No nosso estudo, fatores como a limitações em decorrência da diabetes, em qualquer nível, mostrou-se capaz de influenciar também negativamente a autopercepção de saúde, assim como aqueles que procuraram o serviço de emergência 30,5\% (IC95\%:21,4-41,5) e passaram por internações hospitalares 20,80\% (IC95\%:14,2- 29,3) nos últimos 12 meses em decorrência da Diabetes. Achados estes que corroboram com outros estudos onde descrevem que pacientes que possuem doenças crônicas como a diabetes, acabam por acessar mais os serviços de saúde, fato este, que acaba por influenciar na forma em que os indivíduos percebem sua saúde (Gerhardt,et.al., 2013,Caetano, et.al., 2013).

Observamos neste estudo, que os diabéticos acometidos por multimorbidades possuem limitações e dependem muito dos serviços de saúde, tendem a avaliar negativamente sua percepção de saúde. Tendo em vista este quadro, pacientes que possuem modificações significativas em suas vidas devido às doenças, possuem uma qualidade de vida menor e uma consequente autopercepção negativa de saúde (Gerhardt,et.al, 2013, Idler \& Cartwright,2018). 
Conviver com doenças crônicas como a diabetes pode trazer aos usuários manifestações de descompensação da doença, levando a procura pelos serviços de saúde, seja na emergência em casos agudos ou em internações hospitalares nos casos crônicos ou em que o controle dos sintomas se faz necessário. Pacientes que passam por atendimentos sucessivos nestes serviços no período de 12 meses, obtiveram uma pior avaliação da percepção de saúde. Alguns estudos já descrevem que utilizar a avaliação da autopercepção de saúde pode prever as hospitalizações e a procura por serviços especializados dos usuários (Caetano,et.al, 2013, Nilsson \& Orwelius,2016).

A autopercepção de saúde faz parte de um conjunto de avaliações e relato da saúde individual de cada usuário. Nela são incluídas a avaliação dos sintomas da doença, habilidades funcionais e status de saúde. Sua avaliação pode auxiliar no diagnóstico e tratamento de uma doença, ajudando a esclarecer fatores subjetivos que ajudam a compreender as necessidades de cada paciente, contribuindo para uma intervenção mais direcionada e assertiva, auxiliando na avaliação terapêutica em conjunto com outros dados objetivos como, os diagnósticos de imagem e laboratoriais, já vem sendo descrito na literatura(Teo, et.al., 2016)

Estudos que trazem dados populacionais referentes a DCNT, como a diabetes mostram-se cada vez mais necessários em decorrência de sua crescente demanda. O envelhecimento da população, aumento da expectativa de vida, assim como aumento do número de doenças crônicas corroboram para a criação de políticas públicas que consigam atender a esta população, melhorando os serviços de saúde no país.

\section{Conclusão}

A autopercepção de saúde mostrou-se influenciada pela presença de três ou mais doenças crônicas, assim como pela presença de outras doenças crônicas além da diabetes. A procura pelos serviços de saúde em decorrência da doença nos últimos 12 meses e as limitações que a diabetes pode causar, também aparecem nos relatos dos usuários diabéticos que avaliaram pior sua saúde.

Na literatura, a autopercepção de saúde já havia sido descrita como um importante prognosticador de mortalidade e sugerida como preditora de consultas especializadas e hospitalizações. Nosso estudo sugere ainda, que a autopercepção de saúde possa prever piora no desfecho de saúde dos pacientes, sendo um fator que auxilia na previsão de procura pelos serviços de saúde, possibilitando uma contabilização de possíveis atendimentos em consultas e hospitalizações.

Sugerimos também, a hipótese de que a autopercepção de saúde possa ser incluída na avaliação geral do usuário durante as consultas de enfermagem e médica. Com isso, adicionamos à percepção individual do usuário sobre sua saúde, doença e terapêutica, auxiliando no atendimento e nos cuidados prestados. Cada vez mais, os profissionais de saúde trabalham para oferecer ao usuário maior autonomia e conhecimento sobre seu estado de saúde, sendo a autopercepção de saúde um importante parâmetro que pode auxiliar cada vez mais neste processo.

Neste estudo, mostramos a importância da autopercepção de saúde no contexto de doentes crônicos, relatando os fatores que contribuem para uma pior avaliação de saúde, e de que forma podemos inseri-la na consulta de enfermagem prestada aos usuários. Avaliar a percepção de saúde, pode se tornar uma forma de contemplar o usuário como um todo, auxiliando em uma melhor assistência ao cuidado prestado.

Cada vez mais, utilizamos a troca de experiências e conhecimentos das diferentes áreas da saúde nos atendimentos aos pacientes. A inclusão da interdisciplinaridade nos atendimentos, possibilita isto, preservando a autonomia e integridade de cada método de tratamento das diferentes áreas da saúde, contemplando todas as demandas trazidas pelos usuários.

A autopercepção de saúde contribui para a promoção e prevenção da saúde, e pareceu prever as hospitalizações em decorrência de complicações da doença, neste caso a diabetes. Este parâmetro pode ser utilizado pelos profissionais de saúde, 
visando melhorar a adesão aos tratamentos e controle da doenças crônicas, sendo um importante fator na avaliação dos usuários durante suas consultas de saúde.

Em relação às limitações encontradas no estudo, podemos destacar o tipo de estudo escolhido para o desenvolvimento da pesquisa, como um estudo transversal ele nos possibilitou apenas um recorte momentâneo da população, sem um acompanhamento durante um período de tempo. Outra importante limitação que podemos alencar, foi que por tratar-se de um inquérito populacional com questões fechadas, os entrevistadores não tiveram a oportunidade de registrar detalhes mais importantes sobre os entrevistados.

Estudos que avaliam a autopercepção de saúde, são cada vez mais necessários para a inserção deste parâmetro na avaliação do cuidado, contribuindo para um aperfeiçoamento dos profissionais da saúde. Utilizar este parâmetro durante a avaliação do paciente, pode auxiliar na interdisciplinaridade do cuidado, possibilitando uma transição de cuidado mais completa. Trabalhar com a autopercepção do paciente, permite que ele exponha seu ponto de vista, corroborando para a elaboração de um plano terapêutico direcionado e humanizado.

Sugerimos a realização de mais estudos referente a área de autopercepção de saúde, possibilitando o acompanhamento e avaliações periódicas dos usuários, para melhor observar as diferenças entre as percepções de saúde ao longo dos tratamentos e acompanhamentos em saúde.

\section{Agradecimentos}

Agradecimento à toda equipe da pesquisa PNAUM.

\section{Referências}

ADA. (2017) American Diabetes Association. Standards of medical care in diabetes. Diabetes Care.40(Suppl 1):S1-131. https://care.diabetesjournals.org/content/diacare/suppl/2016/12/15/40.Supplement_1.DC1/DC_40_S1_final.pdf

Agostinho M, Oliveira M, Pinto M, Balardin G \& Harzheim E.(2010). Autopercepção da saúde entre usuários da Atenção Primária em Porto Alegre, RS. Revista Brasileira de Medicina de Família e Comunidade. mar.; 5(17):9-15. Doi:10.5327/Z1809-59092010000500003

Álvares J., Alves M. C. G. P., Escuder M. M. L., Almeida A. M., Izidoro J. B., Junior A. A. G. \& et al (2017). Pesquisa Nacional sobre Acesso, Utilização e Promoção do Uso Racional de Medicamentos: métodos. Rev Saúde Pública. 51 Supl 2:4s. Doi:10.11606/S1518-8787.2017051007027 1

Arruda G. O., Santos A. L., Ferraz E. T., Mantelo H. P. C., Trindade C. A. R. \& Silva S. M. (2015). Associação entre autopercepção de saúde e características sociodemográficas com doenças cardiovasculares em indivíduos adultos. Revista da Escola de Enfermagem da USP,2015.49(1):61-68.Doi: 10.1590/S0080623420150000100008

Boccolini P.de M. M., Duarte C. M. R., Marcelino M. A. \& Boccolini C. S. (2017). Desigualdades sociais nas limitações causadas por doenças crônicas e deficiências no Brasil: Pesquisa Nacional de Saúde - 2013. Ciência \& Saúde Coletiva, 22(11):3537-3546. Doi: 10.1590/1413-812320172211.22552017

Borges A. M, Santos G., Kummer J. A., Fior L., Dal Molin V. \& Wibelinger L.M. (2014). Autopercepção de saúde em idosos residentes em um município do interior do Rio Grande do Sul. Rev. Bras. Geriatr. Gerontol., Rio de Janeiro, 17(1):79-86.Doi: 10.1590/S1809-98232014000100009

BRASIL. (2016) Ministério da saúde. Secretaria da Ciência, Tecnologia e Insumos Estratégicos. Componente populacional: introdução, métodos e instrumentos.: Ministério da Saúde, Brasília.. Caderno 1 - $\quad$ série PNAUM. https://bvsms.saude.gov.br/bvs/publicacoes/componente_populacional_introducao_metodo_instrumentos.pdf

BRASIL. (2018). Ministério da Saúde. Vigilância de fatores de risco e proteção para doenças crônicas por inquérito telefônico. Brasília: Secretaria de Vigilância Epidemiológica. https://portalarquivos2.saude.gov.br/images/pdf/2019/julho/25/vigitel-brasil-2018.pdf

Caetano S.C, Silva C.M.F.P. \& Vettore M.V. (2013). Gender differences in the association of perceived social support and social network with self-rated health status among older adults: a population-based study in Brazil. BMC Geriatrics, 13:122.Doi: 10.1186/1471-2318-13-122.

Cavalcanti G, Doring M, Portella MR, Bortoluzzi EC, Mascarelo A, \& Dellani MP. (2017). Multimorbidade associado à polifarmácia e autopercepção negativa de saúde. Rev. Bras. Geriatr. Gerontol., Rio de Janeiro, 20(5): 635-643.Doi: 10.1590/1981-22562017020.170059

Chiu C.J., Tseng Y.H., Hsu Y.C. \& Shang W.T. (2017) Depressive symptom trajectories in the first 10 years of diabetes diagnosis: antecedent factors and link with future disability in Taiwan. Soc Psychiatry Psychiatr Epidemiol 52: 829.Doi: 10.1007/s00127-016-1314-4.

Cislaghi B \& Cislaghi C. (2019). Self-rated health as a valid indicator for health-equity analyses: evidence from the Italian health interview survey. BMC Public Health 19:533..doi: 10.1186/s12889-019-6839-5 
Dias OV, Chagas RB, Gusmão BM, Pereira FS, Costa SM, Costa FM \& et al. (2016). Prevalência de Diabetes Mellitus. Rev Bras Promoç Saúde, Fortaleza, 29(3): 406-413, jul./set.Doi: 10.1590/1980-5497201700010002

Fabbri E, Zoli M, Gonzalez-Freire M, Salive ME, Studenski AS \& Ferrucci L. (2015). Aging and multimorbidity: new tasks, priorities, and frontiers for integrated gerontological and clinical research. J Am Med Dir Assoc 16(8):640-647.Doi: 10.1016/j.jamda.2015.03.013

Gadelha C.A.G., Costa K.S., Junior J.M.N., Soeiro O.M., Mengue S.S, Motta M.L., Carvalho A.C.C. (2016). PNAUM: abordagem integradora da Assistência Farmacêutica, Ciência, Tecnologia e Inovação. Rev. Saúde Pública. Jul, 50( Suppl 2 ): 3s.Doi: 10.1590/S1518-8787.2016050006153

Gerhardt TE, Kalsing A, Santos VCF, Roese A \& Ruiz ENF.(2013) Perfil de internações por doenças crônicas não transmissíveis sensíveis à atenção primária em idosos da metade sul do RS. Rev Gaúch Enferm. 34(3):124-31.Doi: 10.1590/S1983-14472013000300016

Guariguata L, Whiting D.R., Hambleton I, Beagley J, Linnekamp U \& Shaw J.E.(2014). Global estimates of diabetes prevalence for 2013 and projections for 2035. Diabetes Res Clin Pract 03(2): 137-49.Doi: 10.1016/j.diabres.2013.11.002

IBGE. (2013). PNAD contínua trimestral. Indicadores IBGE: Pesquisa Nacional por Amostra de Domicílios Contínua, Primeiro trimestre 2019. Instituto Brasileiro de Geografia e Estatistica, IBGE - Coordenação de Trabalho e Rendimento.Rio de Janeiro. https://www.ibge.gov.br/estatisticas/multidominio/cultura-recreacao-e-esporte/17270-pnad-continua.html

IDF Atlas.(2017). International Diabetes Federation, 8th edn. Brussels, Belgium: International Diabetes Federation. https://diabetesatlas.org/upload/resources/previous/files/8/IDF_DA_8e-EN-final.pdf

Idler E \& Cartwright K. (2018). What Do We Rate When We Rate Our Health? Decomposing Age-related Contributions to Self-rated Health. Journal of Health and Social Behavior, Vol. 59(1) 74-93 Doi: 10.1177/0022146517750137.

Iser B.P.M., Stopa S.R., Chueiri P.S., Szwarcwald C.L., Malta D.C., Monteiro H.O.C, Duncan B.B \& Schmidt M.I.(2013). Prevalência de diabetes autorreferido no Brasil: resultados da Pesquisa Nacional de Saúde. Epidemiol. Serv. Saúde 24( 2 ): 305-314.Doi: 10.5123/S1679-49742015000200013

Junior A.G.S, Pedro J.O., Oliveira M.C., Furlan M.C.R., Nascimento F.G., Bassler T.C., Vaz E.S.A \& Barcelos L.(2018). Caracterização sociodemográfica e a autopercepção das condições de saúde de idosos. Rev enferm UFPE on line., Recife, 12(3):692-700. https://pesquisa.bvsalud.org/portal/resource/pt/biblio967134

Koche, J. C. (2011). Fundamentos de metodologia científica. Petrópolis: Vozes. http://www.brunovivas.com/wpcontent/uploads/sites/10/2018/07/K\%C3\%B6che-Jos\%C3\%A9-Carlos0D0AFundamentos-de-metodologia-cient\%C3\%ADfica-_teoriada0D0Aci\%C3\%AAncia-e-inicia\%C3\%A7\%C3\%A3o-\%C3\%A0-

pesquisa.pdfhttps://repositorio.ufsm.br/bitstream/handle/1/15824/Lic_Computacao_Metodologia-Pesquisa-Cientifica.pdf?sequence=1

Lefèvre T, D'ivernois J.F., De Andrade V., Crozet C., Lombrail P. \& Gagnayre R. (2014). What do we mean by multimorbidity? an analysis of the literature on multimorbidity measures, associated factors, and impact on health services organization. Rev Epidemiol Santé Publique, 62(5):305-14.Doi: 10.1016/j.respe.2014.09.00

Medeiros S.M., Silva L.S.R., Carneiro J.A., Ramos G.C.F., Barbosa A.T.F. \& Caldeira A. P.(2016). Fatores associados à autopercepção negativa da saúde entre idosos não institucionalizados de Montes Claros, Brasil. Ciênc. Saúde Coletiva. nov.; 2(11):3377-3386. Doi: 10.1590/1413-812320152111.18752015

Mengue S.S, Bertoldi A.D, Boing A.C, Tavares N.U.L, Dal Pizzol T.S, Oliveira M.A., Arrais P.S.D, Ramos L.R, Farias M.R, Luiza V.L., Bernal R.T.I.B. \& Barros A. J. D.(2016). Pesquisa Nacional sobre Acesso, Utilização e Promoção do Uso Racional de Medicamentos (PNAUM): métodos do inquérito domiciliar. Rev Saúde Pública, 50(supl2):4.Doi: 10.1590/S1518-8787.2016050006156

Nilsson E.,Orwelius L. \& Kristenson M.(2016). Patient-reported outcomes in the Swedish National Quality Registers. Journal of Internal Medicine, 279; 14115. Doi: 10.1111/joim.12409

Pereira A. S. et al. (2018). Metodologia da pesquisa científica. [free e-book]. Santa Maria/RS. Ed. UAB/NTE/UFSM. https://repositorio.ufsm.br/bitstream/handle/1/15824/Lic_Computacao_Metodologia-Pesquisa-Cientifica.pdf?sequence=1

Rodrigues R.A.S., Teodózio M.M., Espinosa M.M., Fett W.C.R., Melo C.D. \& Fett C.A. (2018). Timed up and go test and self-perceived health in elderly: population-based study. Rev. bras. cineantropom. desempenho hum. 20( 3 ): 247-257. Doi: 10.5007/1980-0037.2018v20n3p247

Silva J.V.F.; Silva E.C, Rodrigues A.P.R.A \& Miyazawa A.P.(2015). A Relação entre o envelhecimento populacional e as doenças crônicas não transmissíveis: sério desafio de saúde Ciências Biológicas e da Saúde. Maceió, v. 2, n.3, p. 91-100. https://periodicos.set.edu.br/fitsbiosaude/article/view/2079

Teo C.R.P.A., Taglietti R.L., Busato M.A. \& Signor B.(2016) Autopercepção e necessidades de saúde: recurso para enfrentar vulnerabilidades e reorganizar a atenção. Espaço para a saúde - Revista de saúde pública do paraná, Londrina, dezembro, Vol. 17.Doi: 10.22421/15177130-2016v17n2p178

Vejem M., Bjorner J., Bestle M., Lindhardt A. \& Jensen J. (2017). Self-Rated Health as a Predictor of Death after Two Years: The Importance of Physical and Mental Wellbeing Postintensive Care. BioMed Research International, London, 5192640. Doi:10.1155/2017/5192640

WHO (2018) NCD. Noncommunicable Diseases Country Profiles 2018. Geneva: World Health Organization, 2018. Licence: CC BY-NC-SA 3.0 IGO.ISBN 978-92-4-151462-0. Pág. 51 Brazil. https://apps.who.int/iris/handle/10665/274512. 